УДК $517.55+512.718$

\title{
On Convergence of Mellin-Barnes Integrals Representing Solutions of General Algebraic Systems of 3 Equations with 3 Variables
}

\author{
Artem V.Senashov* \\ Institute of Mathematics and Computer Science \\ Siberian Federal University \\ Svobodny, 79, Krasnoyarsk, 660041
}

Russia

Received 29.05.2016, received in revised form 10.11.2016, accepted 06.02.2017

We consider the Mellin-Barnes integral that corresponds to a monomial function of a solution to a system of $n$ algebraic equations in $n$ variables. For $n=3$ we prove that a known necessary condition for convergence for the Mellin-Barnes integral is also sufficient.

Keywords: algebraic equations, Mellin-Barnes integral, convergence.

DOI: 10.17516/1997-1397-2017-10-3-339-344.

\section{Introduction and preliminaries}

In 1921 H. Mellin [1] obtained an integral formula (in the form of Mellin-Barnes integral) for solution of general (universal) algebraic equation. He noted that integral representing the solution has a non-empty domain of convergence. The full convergence domain was found in 2007 in the article of I. A. Antipova [2]. In [3] of V. A. Stepanenko received the integral of MellinBarnes type formally representing the solution of a general system of $n$ polynomial equations with $n$ variables (see also [4]). Recently V. R. Kulikov [5] showed, that for $n>1$ this integral can be really formal, i.e. it can have an empty domain of convergence. He has been found a necessary condition for convergence of the integral, and as a hypothesis he claimed that this condition is also sufficient for the convergence.

The purpose of this work is to prove sufficiency of condition for the convergence of MellinBarnes integral representing the solution of a system of three equations with three variables.

Let us consider a reduced general system of three algebraic equations

$$
\left\{\begin{array}{c}
y_{1}^{m_{1}}+\sum_{i=1}^{p_{1}} x_{i}^{(1)} y_{1}^{\alpha_{i}^{(1)}} y_{2}^{\beta_{i}^{(1)}} y_{3}^{\gamma_{i}^{(1)}}-1=0 \\
y_{2}^{m_{2}}+\sum_{j=1}^{p_{2}} x_{j}^{(2)} y_{1}^{\alpha_{j}^{(2)}} y_{2}^{\beta_{j}^{(2)}} y_{3}^{\gamma_{j}^{(2)}}-1=0 \\
y_{3}^{m_{3}}+\sum_{k=1}^{p_{3}} x_{k}^{(3)} y_{1}^{\alpha_{k}^{(3)}} y_{2}^{\beta_{k}^{(3)}} y_{3}^{\gamma_{k}^{(3)}}-1=0
\end{array}\right.
$$

where $x_{1}^{(1)}, \ldots, x_{p_{1}}^{(1)}, x_{1}^{(2)}, \ldots, x_{p_{2}}^{(2)}, x_{1}^{(3)}, \ldots, x_{p_{3}}^{(3)}$ are variable complex coefficients. Consider the vector $x=\left(x^{(1)}, x^{(2)}, x^{(3)}\right)$, where $x^{(i)}=\left(x_{1}^{(i)}, \ldots, x_{p_{i}}^{(i)}\right)$ are the coefficients of the $i$-equation,

*asenashov@mail.ru

(c) Siberian Federal University. All rights reserved 
$i=1,2,3$. Similarly, we introduce vectors

$$
\begin{aligned}
& \alpha=\left(\alpha_{1}^{(1)}, \ldots, \alpha_{p_{1}}^{(1)}, \alpha_{1}^{(2)}, \ldots, \alpha_{p_{2}}^{(2)}, \alpha_{1}^{(3)}, \ldots, \alpha_{p_{3}}^{(3)}\right), \\
& \beta=\left(\beta_{1}^{(1)}, \ldots, \beta_{p_{1}}^{(1)}, \beta_{1}^{(2)}, \ldots, \beta_{p_{2}}^{(2)}, \beta_{1}^{(3)}, \ldots, \beta_{p_{3}}^{(3)}\right), \\
& \gamma=\left(\gamma_{1}^{(1)}, \ldots, \gamma_{p_{1}}^{(1)}, \gamma_{1}^{(2)}, \ldots, \gamma_{p_{2}}^{(2)}, \gamma_{1}^{(3)}, \ldots, \gamma_{p_{3}}^{(3)}\right) .
\end{aligned}
$$

Solution $y(x)=\left(y_{1}(x), y_{2}(x), y_{3}(x)\right)$ of this system of equations is an algebraic vector-function of $x$. In the article [3] for monomial function $y_{1}^{\mu_{1}} y_{2}^{\mu_{2}} y_{3}^{\mu_{3}}$ of solution $y(x)$ of system (1) was found formal (without justification of convergence) integral representation in the form of Mellin-Barnes integral:

$$
y_{1}^{\mu_{1}} y_{2}^{\mu_{2}} y_{3}^{\mu_{3}}(x)=\frac{1}{(2 \pi i)^{3}} \int_{\delta+i R^{p_{1}+p_{2}+p_{3}}} F(s) x^{-s} d s .
$$

Here $x^{-s}$ is the kernel of the inverse Mellin transform, $F(s)$ is a meromorphic function

$$
\frac{\prod_{k=1}^{3} \prod_{j=1}^{p_{k}} \Gamma\left(s_{j}^{(k)}\right) \Gamma\left(\frac{\mu_{1}}{m_{1}}-\frac{1}{m_{1}}\langle\alpha, s\rangle\right) \Gamma\left(\frac{\mu_{2}}{m_{2}}-\frac{1}{m_{2}}\langle\beta, s\rangle\right) \Gamma\left(\frac{\mu_{3}}{m_{3}}-\frac{1}{m_{3}}\langle\gamma, s\rangle\right) Q(s)}{\Gamma\left(\frac{\mu_{1}}{m_{1}}-\frac{1}{m_{1}}\langle\alpha, s\rangle+\left|s^{(1)}\right|+1\right) \Gamma\left(\frac{\mu_{2}}{m_{2}}-\frac{1}{m_{2}}\langle\beta, s\rangle+\left|s^{(2)}\right|+1\right) \Gamma\left(\frac{\mu_{3}}{m_{3}}-\frac{1}{m_{3}}\langle\gamma, s\rangle+\left|s^{(3)}\right|+1\right)}
$$

with a some polynomial $Q(s)$, the vector $\delta \in R^{p_{1}+p_{2}+p_{3}}$ is chosen such that the set of integration $\delta+i R^{p_{1}+p_{2}+p_{3}}$ does not contain the poles of $\Gamma$-function from numerator of $F(s)$.

For every triple of indices $(i, j, k) \in\left\{1, \cdots, p_{1}\right\} \times\left\{1, \cdots, p_{2}\right\} \times\left\{1, \cdots, p_{3}\right\}$ we will form a $3 \times 3$ matrix

$$
A_{i j k}=\left(\begin{array}{ccc}
\alpha_{i}^{(1)} & \alpha_{j}^{(2)} & \alpha_{k}^{(3)} \\
\beta_{i}^{(1)} & \beta_{j}^{(2)} & \beta_{k}^{(3)} \\
\gamma_{i}^{(1)} & \gamma_{j}^{(2)} & \gamma_{k}^{(3)}
\end{array}\right)
$$

In other words, we consider all $3 \times 3$ matrices in which $q$-th column consist of the exponents of the monomials of $q$-th equation.

As mentioned above the purpose of this work is to prove the following theorem, whose statement was made as a hypothesis by V. R. Kulikov: Under the main minor of matrix we understand a minor, which involves the rows and columns with the same set of indices.

Theorem. The integral (2) has non-empty domain of convergence if and only if all the main minors of every matrix $A_{i j k}$ are positive, i.e.

$$
\begin{aligned}
& \alpha_{i}^{(1)}>0, \beta_{j}^{(2)}>0, \gamma_{k}^{(3)}>0, \\
& \alpha_{i}^{(1)} \beta_{j}^{(2)}-\alpha_{j}^{(2)} \beta_{i}^{(1)}>0, \beta_{j}^{(2)} \gamma_{k}^{(3)}-\beta_{k}^{(3)} \gamma_{j}^{(2)}>0, \alpha_{i}^{(1)} \gamma_{k}^{(3)}-\alpha_{k}^{(3)} \gamma_{i}^{(1)}>0, \\
& \alpha_{i}^{(1)} \beta_{j}^{(2)} \gamma_{k}^{(3)}+\alpha_{j}^{(2)} \beta_{k}^{(3)} \gamma_{i}^{(1)}+\beta_{i}^{(1)} \gamma_{j}^{(2)} \alpha_{k}^{(3)}-\alpha_{k}^{(3)} \beta_{j}^{(2)} \gamma_{i}^{(1)}-\alpha_{j}^{(2)} \beta_{i}^{(1)} \gamma_{k}^{(3)}-\alpha_{i}^{(1)} \beta_{k}^{(3)} \gamma_{j}^{(2)}>0 .
\end{aligned}
$$

\section{Proof of the main result}

Recall that the necessity of condition (3) has been proven by V. R. Kulikov in [5]. Here we prove sufficiency of them.

Note, that in (2) the integration is performed on the imaginary subspace of variables $s$. We write $s$ in the form $s=u+i v$; thus, we can suppose, that the integration in (2) is carried out by the variables $v=\left(v_{1}^{(1)}, \ldots, v_{p_{1}}^{(1)}, v_{1}^{(2)}, \ldots, v_{p_{2}}^{(2)}, v_{1}^{(3)}, \ldots, v_{p_{3}}^{(3)}\right)$. 
Consider the following function which is composed by arguments of $\Gamma$-function from the numerator of $F(u)$ :

$$
\begin{aligned}
g(v) & =\sum_{i=1}^{p_{1}}\left|v_{i}^{(1)}\right|+\left|\frac{1}{m_{1}}\langle\alpha, v\rangle\right|-\left|\sum_{i=1}^{p_{1}} v_{i}^{(1)}-\frac{1}{m_{1}}\langle\alpha, v\rangle\right|+ \\
& +\sum_{j=1}^{p_{2}}\left|v_{j}^{(2)}\right|+\left|\frac{1}{m_{2}}\langle\beta, v\rangle\right|-\left|\sum_{j=1}^{p_{2}} v_{j}^{(2)}-\frac{1}{m_{2}}\langle\beta, v\rangle\right|+ \\
& +\sum_{k=1}^{p_{3}}\left|v_{k}^{(3)}\right|+\left|\frac{1}{m_{3}}\langle\gamma, v\rangle\right|-\left|\sum_{k=1}^{p_{3}} v_{k}^{(3)}-\frac{1}{m_{3}}\langle\gamma, v\rangle\right| .
\end{aligned}
$$

The general scheme for computation of the convergence domain for Mellin-Barnes integral is given by Theorem of Nilsson, Passare and Tsikh [6]. Applying to the integral (2) this theorem is formulated as follows.

For any integration set $\delta+i R^{p_{1}+p_{2}+p_{3}}$ which does not contain singularities of the integrand the domain of convergence of the Mellin-Barnes integral has the form $\operatorname{Arg}^{-1}(U)$, where

$$
U=\bigcap_{\|v\|=1}\left\{\theta \in R^{p_{1}+p_{2}+p_{3}}:|\langle v, \theta\rangle|<\frac{\pi}{2} g(v)\right\},
$$

where Arg is a mapping $\left(x_{1}^{(1)}, \cdots, x_{p_{3}}^{(1)}\right) \rightarrow\left(\arg x_{1}^{(1)}, \cdots, \arg x_{p_{3}}^{(3)}\right)$.

Note that $g(v) \geqslant 0$. One can easily prove the following statement.

Lemma. The function $g(v)=0$ if and only if the elements from each of the following three groups have the same sign:

$$
\begin{aligned}
& \left\{v_{i}^{(1)}, i=1, \ldots, p_{1}\right\},-\frac{1}{m_{1}}\langle\alpha, v\rangle, \\
& \left\{v_{j}^{(2)}, j=1, \ldots, p_{2}\right\},-\frac{1}{m_{2}}\langle\beta, v\rangle, \\
& \left\{v_{k}^{(3)}, k=1, \ldots, p_{3}\right\},-\frac{1}{m_{3}}\langle\gamma, v\rangle .
\end{aligned}
$$

The function $g(v)$ is continuous and therefore it arrives the minimum value on the sphere $\|v\|=1$. Consequently the domain (5) is non-empty if and only if $g(v)>0$ for all $v$ from the sphere. Thus, we need to show, that under the condition of positivity of the main minors (3) the conclusion of the lemma holds only in the case $v=0$.

We divide the scalar products, which participate in the expression for $g(v)$, into three parts

$$
\begin{aligned}
& \langle\alpha, v\rangle=\left\langle\alpha^{(1)}, v^{(1)}\right\rangle+\left\langle\alpha^{(2)}, v^{(2)}\right\rangle+\left\langle\alpha^{(3)}, v^{(3)}\right\rangle, \\
& \langle\beta, v\rangle=\left\langle\beta^{(1)}, v^{(1)}\right\rangle+\left\langle\beta^{(2)}, v^{(2)}\right\rangle+\left\langle\beta^{(3)}, v^{(3)}\right\rangle, \\
& \langle\gamma, v\rangle=\left\langle\gamma^{(1)}, v^{(1)}\right\rangle+\left\langle\gamma^{(2)}, v^{(2)}\right\rangle+\left\langle\gamma^{(3)}, v^{(3)}\right\rangle,
\end{aligned}
$$

where $\alpha^{(i)}=\left(\alpha_{1}^{(i)}, \ldots, \alpha_{p_{i}}^{i}\right), \beta^{(i)}=\left(\beta_{1}^{(i)}, \ldots, \beta_{p_{i}}^{i}\right), \gamma^{(i)}=\left(\gamma_{1}^{(i)}, \ldots, \gamma_{p_{i}}^{i}\right)$.

In the space $R^{p_{1}+p_{2}+p_{3}}$ consider eight orthants of the kind $K_{\varepsilon}=K_{\varepsilon_{1}} \times K_{\varepsilon_{2}} \times K_{\varepsilon_{3}}$, where $\varepsilon_{k}$ are the signs,+- and $K_{\varepsilon_{k}}$ are the orthants $R_{+}^{p_{k}}$ or $R_{-}^{p_{k}}$. Elements $v^{(k)} \in K_{\varepsilon_{k}}$ are presented in the form $\tau^{(k)} t^{(k)}$, where $\tau^{(k)}$ runs part of the unit sphere $\left(\|v\|^{(k)}=1\right) \cap R_{+}^{p_{k}}, t^{(k)} \in R_{ \pm}$in depending on $\varepsilon_{k}$. If for given vector $v \in R^{p_{1}+p_{2}+p_{3}}$ conclusion of the lemma is valid, then $v$ belongs to one of orthants $K_{\varepsilon}$. 
Scalar products on $K_{\varepsilon}$ introduced above can be written as

$$
\begin{aligned}
& \langle\alpha, v\rangle=\left\langle\alpha^{(1)}, \tau^{(1)} t^{(1)}\right\rangle+\left\langle\alpha^{(2)}, \tau^{(2)} t^{(2)}\right\rangle+\left\langle\alpha^{(3)}, \tau^{(3)} t^{(3)}\right\rangle, \\
& \langle\beta, v\rangle=\left\langle\beta^{(1)}, \tau^{(1)} t^{(1)}\right\rangle+\left\langle\beta^{(2)}, \tau^{(2)} t^{(2)}\right\rangle+\left\langle\beta^{(3)}, \tau^{(3)} t^{(3)}\right\rangle, \\
& \langle\gamma, v\rangle=\left\langle\gamma^{(1)}, \tau^{(1)} t^{(1)}\right\rangle+\left\langle\gamma^{(2)}, \tau^{(2)} t^{(2)}\right\rangle+\left\langle\gamma^{(3)}, \tau^{(3)} t^{(3)}\right\rangle .
\end{aligned}
$$

Take out $t^{(k)}$ from the sign of the scalar product

$$
\begin{aligned}
& \left\langle\alpha^{(1)}, \tau^{(1)}\right\rangle t^{(1)}+\left\langle\alpha^{(2)}, \tau^{(2)}\right\rangle t^{(2)}+\left\langle\alpha^{(3)}, \tau^{(3)}\right\rangle t^{(3)}, \\
& \left\langle\beta^{(1)}, \tau^{(1)}\right\rangle t^{(1)}+\left\langle\beta^{(2)}, \tau^{(2)}\right\rangle t^{(2)}+\left\langle\beta^{(3)}, \tau^{(3)}\right\rangle t^{(3)}, \\
& \left\langle\gamma^{(1)}, \tau^{(1)}\right\rangle t^{(1)}+\left\langle\gamma^{(2)}, \tau^{(2)}\right\rangle t^{(2)}+\left\langle\gamma^{(3)}, \tau^{(3)}\right\rangle t^{(3)} .
\end{aligned}
$$

We introduce the notation $\left\langle\alpha^{(i)}, \tau^{(i)}\right\rangle=\alpha_{i},\left\langle\beta^{(i)}, \tau^{(i)}\right\rangle=\beta_{i},\left\langle\gamma^{(i)}, \tau^{(i)}\right\rangle=\gamma_{i}$. Then the scalar products takes the following form

$$
\begin{aligned}
& \alpha_{1} t^{(1)}+\alpha_{2} t^{(2)}+\alpha_{3} t^{(3)}, \\
& \beta_{1} t^{(1)}+\beta_{2} t^{(2)}+\beta_{3} t^{(3)}, \\
& \gamma_{1} t^{(1)}+\gamma_{2} t^{(2)}+\gamma_{3} t^{(3)}
\end{aligned}
$$

We will prove that under the conditions (3) all main minors of the matrix of the linear transformation (7) are positive:

$$
\begin{aligned}
& \alpha_{1}>0, \beta_{2}>0, \gamma_{3}>0, \\
& \alpha_{1} \beta_{2}-\alpha_{2} \beta_{1}>0, \beta_{2} \gamma_{3}-\beta_{3} \gamma_{2}>0, \alpha_{1} \gamma_{3}-\alpha_{3} \gamma_{1}>0, \\
& \alpha_{1} \beta_{2} \gamma_{3}+\alpha_{2} \beta_{3} \gamma_{1}+\alpha_{3} \beta_{1} \gamma_{2}-\alpha_{3} \beta_{2} \gamma_{1}-\alpha_{2} \beta_{1} \gamma_{3}-\alpha_{1} \beta_{3} \gamma_{2}>0 .
\end{aligned}
$$

Notice, that $\alpha_{1}>0, \beta_{2}>0, \gamma_{3}>0$ as scalar products of vectors with positive coordinates on non-zero vectors with non-negative coordinates (recall, that $\tau^{(k)}$ belongs to the unit sphere).

Further, the difference

$$
\alpha_{1} \beta_{2}-\alpha_{2} \beta_{1}=\left\langle\alpha^{(1)}, \tau^{(1)}\right\rangle\left\langle\beta^{(2)}, \tau^{(2)}\right\rangle-\left\langle\alpha^{(2)}, \tau^{(2)}\right\rangle\left\langle\beta^{(1)}, \tau^{(1)}\right\rangle
$$

is equal to bilinear form on $\tau^{(1)}$ and $\tau^{(2)}$ :

$$
\sum_{i, j}\left(\alpha_{i}^{(1)} \beta_{j}^{(2)}-\alpha_{j}(2) \beta_{i}^{(1)}\right) \tau_{i}^{(1)} \tau_{j}^{(2)}
$$

By conditions (3) the coefficients of this form are positive and the coordinates of non-zero vectors $\tau^{(1)}$ and $\tau^{(2)}$ are non-negative, so $\alpha_{1} \beta_{2}-\alpha_{2} \beta_{1}>0$. Similarly, we prove the positiveness of all the remaining minors of the matrix from (8).

Now we are ready to prove, that if under the conditions (3) conclusion of the lemma is valid, then $v=0$.

Consider two cases when the conclusion of the lemma holds.

1. The elements of all the groups from (6) are non-negative. Since $m_{i}$ are positive, in our notation $v^{k}=\tau^{(k)} t^{(k)}$ it means that

$$
\left\{\begin{array}{l}
\alpha_{1} t^{(1)}+\alpha_{2} t^{(2)}+\alpha_{3} t^{(3)} \leqslant 0 \\
\beta_{1} t^{(1)}+\beta_{2} t^{(2)}+\beta_{3} t^{(3)} \leqslant 0 \\
\gamma_{1} t^{(1)}+\gamma_{2} t^{(2)}+\gamma_{3} t^{(3)} \leqslant 0 \\
t^{(1)} \geqslant 0 \\
t^{(2)} \geqslant 0 \\
t^{(3)} \geqslant 0
\end{array}\right.
$$


In the first three inequalities only non-negative elements are presented. Since $\alpha_{1}>0, \beta_{2}>0$, $\gamma_{3}>0$, then equality is reached only in the case $t^{(i)}=0, i=1,2,3$.

2. The elements of the first group from (6) are negative, and the elements of the second and of the third groups are non-positive, i.e.

$$
\left\{\begin{array}{l}
\alpha_{1} t^{(1)}+\alpha_{2} t^{(2)}+\alpha_{3} t^{(3)} \leqslant 0 \\
\beta_{1} t^{(1)}+\beta_{2} t^{(2)}+\beta_{3} t^{(3)} \geqslant 0 \\
\gamma_{1} t^{(1)}+\gamma_{2} t^{(2)}+\gamma_{3} t^{(3)} \geqslant 0 \\
t^{(1)} \geqslant 0 \\
t^{(2)} \leqslant 0 \\
t^{(3)} \leqslant 0
\end{array}\right.
$$

With help of the first line set to zero coefficients of $t^{(1)}$ in the second and in the third inequalities:

$$
\left\{\begin{array}{l}
\alpha_{1} t^{(1)}+\alpha_{2} t^{(2)}+\alpha_{3} t^{(3)} \leqslant 0 \\
\left(\beta_{2} \alpha_{1}-\alpha_{2} \beta_{1}\right) t^{(2)}+\left(\beta_{3} \alpha_{1}-\alpha_{3} \beta_{1}\right) t^{(3)} \geqslant 0 \\
\left(\gamma_{2} \alpha_{1}-\alpha_{2} \gamma_{1}\right) t^{(2)}+\left(\gamma_{3} \alpha_{1}-\alpha_{3} \gamma_{1}\right) t^{(3)} \geqslant 0 \\
t^{(1)} \geqslant 0 \\
t^{(2)} \leqslant 0 \\
t^{(3)} \leqslant 0
\end{array}\right.
$$

By the proving above, the coefficient of $t^{(2)}$ in the second inequality and the coefficient of $t^{(3)}$ in the third inequality became are positive. Denote by $d_{1}, d_{2}, b_{1}$ and $b_{2}$ the coefficients $\left\langle\beta_{2} \alpha_{1}-\alpha_{2} \beta_{1}\right\rangle,\left\langle\beta_{3} \alpha_{1}-\alpha_{3} \beta_{1}\right\rangle,\left\langle\gamma_{2} \alpha_{1}-\alpha_{2} \gamma_{1}\right\rangle$ and $\left\langle\gamma_{3} \alpha_{1}-\alpha_{3} \gamma_{1}\right\rangle$ respectively.

The determinant of the first three inequalities of the system (8) is equal to $\alpha_{1}\left(\begin{array}{ll}d_{1} & b_{2} \\ b_{1} & d_{2}\end{array}\right)$. As $\alpha_{1}\left(\begin{array}{ll}d_{1} & b_{2} \\ b_{1} & d_{2}\end{array}\right)>0$, then $d_{1} b_{2}>b_{1} d_{2}$

If at least one of the quantities $b_{1}, d_{2}$ is positive, then at least one of the 2nd and 3rd inequalities in quadrant $t^{(2)} \geqslant 0, t^{(3)} \geqslant 0$ is valid only in the coordinate origin.

Consider the case $d_{2}<0$ and $b_{1}<0$.

In this case, the half-planes $d_{1} t^{(2)}+d_{2} t^{(3)} \geqslant 0, b_{1} t^{(2)}+b_{2} t^{(3)} \geqslant 0$ cross quadrant $t^{(2)}<0$, $t^{(3)}<0$.

When we construct the domains for the second inequality and for the third inequality in the third quarter of the coordinate it is obtained that the intersection of the domains is the origin-point $(0,0)$, as $\frac{d_{1}}{b_{2}}>\frac{b_{1}}{d_{2}}$.

So, we have proved, that $t^{(2)}=t^{(3)}=0$. The first line under these conditions looks like $\alpha_{1} t^{(1)} \leqslant 0$. By the condition, $t^{(1)} \geqslant 0$ and $\alpha_{1}>0$. Then $t^{(1)}=0$. Thus, in this case only the trivial solution $t^{(1)}=t^{(2)}=t^{(3)}=0$ of the system (8) is posible. The remaining cases of the signs of the elements of the groups (6) are considered similarly.

Returning to the original designations, we find that under the condition $(3) g(v)=0$ if and only if $v=0$. This means that the domain of convergence for Mellin-Barnes integral (2) is not empty.

Thus we proved that the condition (3) is sufficient for the convergence of the integral (2).

Finally, we note, that this result was also obtained independently by V. R. Kulikov. 
This work was supported by a grant from the Russian government to conduct research under the direction of of leading scientists in the Siberian federal university of (contract no. 14.Y26.31.0006) and the Russian Federation President's grant for support of leading scientific schools no. NS-9149.2016.1.

\section{References}

[1] H.Mellin, Résolution de I'équation algébrique générale à l'aide de la fonction $\Gamma$, S.C.R. Acad. Sci., 172(1921), 658-661.

[2] I.A.Antipova, Inversion of many-dimensional Mellin transforms and solutions of algebraic equations, Sbornik Mathematics, 198(2007), no. 4, 447-463.

[3] V.A.Stepanenko, The solution of a system of $n$ algebraic equations in $n$ unknowns by means of hypergeometric functions, Vestnik Krasnoyar. Gosudarst. Univer., 1(2003), 35-48 (in Russian).

[4] I.A.Antipova, An Expression for the Superposition of General Algebraic Functions in Terms of Hypergeometric Series, Siberian Mathematical Journal, 44(2003), no. 5, 757-764.

[5] V.R.Kulikov, Conditions for convergence of the Mellin-Barnes integral for solution to system of algebraic equations, J. Siberian Federal University. Mathematics and Physics, 7(2014), no. $3,339-346$.

[6] T.M.Sadykov, A.K.Tsikh, Hypergeometric and algebraic functions of several variables, Moscow, Nauka, 2014.

\section{О сходимости интеграла Меллина-Барнса, представляющего решение общей алгебраической системы трех уравнений с тремя неизвестными}

Артем В. Сенашов

Институт математики и фундаментальной информатики Сибирский федеральный университет Свободный, 79, Красноярск, 660041

Россия

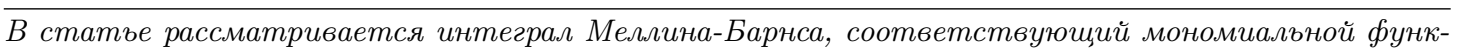
иии решения системы $n$ алгебраических уравнений от $n$ неизвестных. Для $n=3$ показано, что необходимое условие сходимости интеграла Меллина-Барнса является также и достаточным.

Ключевые слова: алгебраические уравнения, интеграл Меллина-Барнса, сходимость. 